\title{
Synchronization properties of coupled semiconductor lasers subject to filtered optical feedback
}

\author{
Miguel C. Soriano, ${ }^{1}$ Flavio Ruiz-Oliveras, ${ }^{1,2}$ Pere Colet, ${ }^{1}$ and Claudio R. Mirasso ${ }^{1}$ \\ ${ }^{1}$ Instituto de Física Interdisciplinar y Sistemas Complejos, IFISC (CSIC-UIB), \\ Campus Universitat Illes Balears, E-07122 Palma de Mallorca, Spain \\ ${ }^{2}$ Centro de Investigaciones en Óptica, Loma del Bosque 115, Lomas del Campestre, 37150 León, Guanajuato, México
}

(Received 27 June 2007; revised manuscript received 22 April 2008; published 30 October 2008)

\begin{abstract}
We study numerically the synchronization properties of two unidirectionally coupled semiconductor lasers subject to filtered optical feedback. By adding a perturbation (a message) to the output of the master laser, we show that mutual information allows distinguishing between chaotic synchronization (at low to moderate coupling strengths) and injection locking (at large coupling strength). We find that a receiver subject to a feedback similar to that of the emitter (closed-loop receiver) shows better synchronization with the master laser when compared with a receiver without feedback (open-loop receiver). Closed-loop receivers also show better capability to recover weak messages. The filter in the feedback loop allows reducing the bandwidth of the chaotic carrier, improves the synchronization with respect to the conventional feedback case, and requires less coupling strength with a minor loss in complexity.
\end{abstract}

DOI: 10.1103/PhysRevE.78.046218

PACS number(s): 05.45.Vx, 05.45.Gg, 42.65.Sf

\section{INTRODUCTION}

Optical feedback is known to create instabilities in the emission of semiconductor lasers (SCLs). In most of the cases, these instabilities manifest in chaotic oscillations of the emitted optical power. While chaotic outputs are usually undesired, it has also been shown that they can be used to encode information and improve the security of data transmission in optical communication systems [1-6]. Successful chaos-based communication is based on synchronization of an emitter master laser (ML) and a receiver slave laser (SL), which is achieved by injecting part of the light emitted by the ML into the SL. In most cases, the chaos is simply generated at the ML by feeding back the light reflected by an external mirror. The SL at the receiver can be subject to a feedback loop (closed-loop configuration) or not (open-loop configuration). In both cases emitter and receiver should have similar devices and operating conditions to synchronize $[5,7,8]$. The open-loop scheme is mechanically more stable, but the closed loop is known to provide better synchronization if the ML and SL external cavities match almost perfectly, otherwise a poor degree of synchronization is observed $[9,10]$. Besides the simple optical feedback scheme, other configurations including optical injection [11], amplified injection [12], amplified optical feedback [13], incoherent optical feedback and injection $[14,15]$ or opto-electronic feedback [16] have also been investigated.

Up to now, the full large bandwidth generated by the emitter has been transmitted to the receiver to achieve synchronization. However, this can easily generate cross talk if wave-division multiplexing is intended. Furthermore, most communication channels suffer from bandwidth limitations, which can degrade the synchronization properties of chaotic systems [17]. Consequently, sources generating chaos with narrower spectra would also be desirable. It is known that the dynamical response of a SCL subject to filtered optical feedback (FOF) can be strongly modified with respect to the conventional feedback case $[18,19]$. This change is more noticeable if the bandwidth of the filter is narrower than the bandwidth of the feedback-induced dynamics in the absence of the filter. While a chaotic output signal can still be generated, the question of whether synchronization occurs is still open. In this paper we explore the effect on the chaos bandwidth and the synchronization when two SCL subject to FOF are coupled unidirectionally.

The injection of the ML field into the SL resembles optical injection schemes that result in injection locking. However, chaos synchronization of two identical SCLs differs from injection locking. Chaos synchronization is robust in front of small perturbations [20], so in the synchronization regime if the output of the ML is subject to a small perturbation the SL reproduces the dynamics of the ML, filtering out the perturbation. When used for chaos-based communications the message plays the role of a small perturbation, and this is precisely the mechanism that allows for the message to be recovered at the receiver. On the contrary, in the injection-locking regime the receiver locks to the incoming field (including any eventual perturbation of the ML output). To distinguish between these two regimes we codify a message (small perturbation) on the output of the ML (carrier) and measure the average mutual information between the output of the SL and the carrier and between the output of the SL and the injected field (carrier with message). In the synchronization regime the former is larger than the latter, while the opposite occurs in the injection locking regime.

\section{RATE EQUATION MODEL}

Figure 1 shows a schematic drawing of two unidirectionally coupled lasers in two possible master-slave configurations, namely, open- and closed-loop schemes. In the conventional open-loop configuration, the SL is a free running laser subject to the input of the ML. However, a second option, although more complicated, could be implemented: a similar grating to that in the ML can be placed in front of the input facet of the SL. In the closed-loop configuration, the ML and SL are both subject to feedback. 
(a)

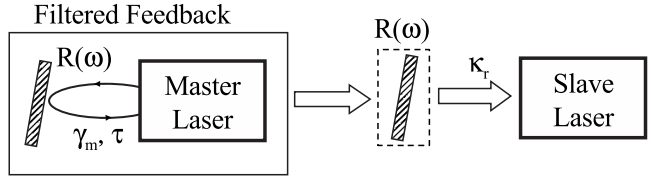

(b) Filtered Feedback

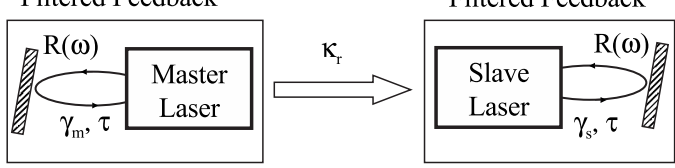

FIG. 1. ML and SL in the (a) open- and (b) closed-loop configuration. In the open-loop configuration, we include the possibility of having an extra filter in front of the SL (see dotted box). $\gamma_{m, s}$ are the feedback strengths, $\tau$ is the feedback delay, $\kappa_{r}$ is the coupling strength, and $R(\omega)$ is the frequency response of the grating.

SCLs subject to FOF can be modelled via rate equations for a slowly varying amplitude of the electric field $E(t)$ and the carrier number $N(t)[18,19]$. The equation for the carrier number in the ML (denoted by $m$ ) and SL (denoted by $s$ ), if we assume single-longitudinal-mode operation, is given by

$$
\dot{N}_{m, s}(t)=\frac{I}{e}-\frac{N_{m, s}(t)}{\tau_{N}}-G_{m, s}(t) P_{m, s}(t),
$$

and the equations for the evolution of the electric field in the ML are

$$
\begin{aligned}
& \dot{E}_{m}(t)=\frac{1+i \alpha}{2}\left(G_{m}(t)-\frac{1}{\tau_{p}}\right) E_{m}(t)+\gamma_{m} F_{m}(t), \\
& \dot{F}_{m}(t)=\Lambda_{m} E_{m}(t-\tau) e^{-i \Phi_{m}}+\left(i \omega_{m}-\Lambda_{m}\right) F_{m}(t),
\end{aligned}
$$

where the optical feedback has been introduced using the Lang-Kobayashi approach [21], which takes into account a single reflection from the external mirror. Furthermore, we consider only one longitudinal mode of the internal cavity and neglect the frequency dependence of the material gain. These approximations limit the range of validity of the model [22], and beyond this range more accurate models including the frequency dependence of the gain [23] or also allowing for longitudinal multimode operation [24] should be used. However, we consider here a regime of low to intermediate feedback strength where the Lang-Kobayashi model is widely used and its predictions have been contrasted with experimental results $[9,10,19,25,26]$.

The equations for the evolution of the electric field in the $\mathrm{SL}$ in the open-loop scheme are

$$
\begin{gathered}
\dot{E}_{s}(t)=\frac{1+i \alpha}{2}\left(G_{s}(t)-\frac{1}{\tau_{p}}\right) E_{s}(t)+\kappa_{r} F_{p}(t), \\
F_{p}(t)=\Lambda_{p} E_{m}(t) e^{-i \Phi_{p}}+\left(i \omega_{p}-\Lambda_{p}\right) F_{p}(t),
\end{gathered}
$$

while in the closed-loop scheme they are

$$
\begin{gathered}
\dot{E}_{s}(t)=\frac{1+i \alpha}{2}\left(G_{s}(t)-\frac{1}{\tau_{p}}\right) E_{s}(t)+\gamma_{s} F_{s}(t)+\kappa_{r} E_{m}(t), \\
\dot{F}_{s}(t)=\Lambda_{s} E_{s}(t-\tau) e^{-i \Phi_{s}}+\left(i \omega_{s}-\Lambda_{s}\right) F_{s}(t) .
\end{gathered}
$$

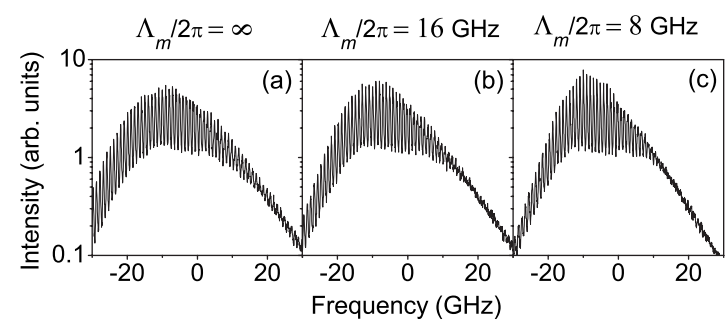

FIG. 2. ML optical spectra for three ML filter widths. Parameters: $\gamma_{m}=25 \mathrm{~ns}^{-1}, I=1.5 I_{\mathrm{th}}$.

The external optical feedback $F_{m, s}(t)$ is filtered by a grating which has a Lorentzian transmission response. $F_{p}(t)$ is the field emitted by the ML, which can also be filtered by a grating prior to the injection into the SL. For a filter of infinite width, which is equivalent to the absence of a filter, $F_{m}(t)=E_{m}(t-\tau), \quad F_{s}(t)=E_{s}(t-\tau)$, and $F_{p}(t)=E_{m}(t) . \quad\left|E_{m, s}\right|^{2}$ $=P_{m, s}$ is the optical power in terms of the number of photons. The terms $\kappa_{r} F_{p}(t)$ and $\kappa_{r} E_{m}(t)$ account for the light that comes from the ML into the SL in the open loop and the closed loop, respectively, $\kappa_{r}$ is the coupling strength, and there is no detuning between ML and SL emission frequencies. The laser parameters are considered to be identical for both devices. $\alpha=5$ is the linewidth enhancement factor. $\tau_{p}$ $=2 \mathrm{ps}$ is the photon lifetime. In the definition of the gain $G_{m, s}=g\left(N_{m, s}-N_{o}\right) /\left(1+s P_{m, s}\right), g=1.5 \times 10^{-8} \mathrm{ps}^{-1}$ is the differential gain, $N_{o}=1.5 \times 10^{8}$ is the carrier number at transparency, and $s=5 \times 10^{-7}$ is the gain compression factor. $I$ denotes the injected current; $I_{\mathrm{th}}=14.7 \mathrm{~mA}$ is the threshold current. $e$ is the electron charge and $\tau_{N}=2$ ns is the carrier lifetime. $\gamma_{m, s}$ are the feedback strengths. The filter half width at half the maximum is $\Lambda_{m, s, p}$ and its detuning, with respect to the free-running emission frequency of the laser, is $\omega_{m, s, p}$. $\tau=1 \mathrm{~ns}$ is the external cavity delay and $\Phi_{m, s}$ are the phases accumulated by the electric field in the external cavity round trips. In the long-cavity limit, like the one we are considering here, the phases do not play significant roles, so we take $\Phi_{m, s, p}=0$ [4]. Without loss of generality, the flying time between ML and SL is taken as 0.

Figures 2(a)-2(c) show the ML optical spectra for filter widths of $\Lambda_{m} / 2 \pi=\infty, 16$, and $8 \mathrm{GHz}$. The feedback dynamics covers the range of approximately -25 to $20 \mathrm{GHz}$. A filter in the feedback loop reduces the frequency width of the optical spectrum. Time series for these three different cases are shown in Fig. 3. Here, filters are centered around the maxima of the optical spectra $(-8 \mathrm{GHz}$ for our parameter values). Note that the optical spectra for conventional and filtered feedback are all shifted to negative frequencies as a consequence of the linewidth enhancement factor (also known as the $\alpha$ factor) [27].

In this work, we estimate the complexity of the system by computing the autocorrelation time. Short autocorrelation times are associated with high complexity, while long autocorrelation times are associated with low complexity. The autocorrelation time $T_{c}$ is defined as [13]

$$
T_{c}=\int_{0}^{\infty} d \tau A^{2}(\tau),
$$

where $A(t)$ is the normalized autocorrelation of the master laser optical power: 


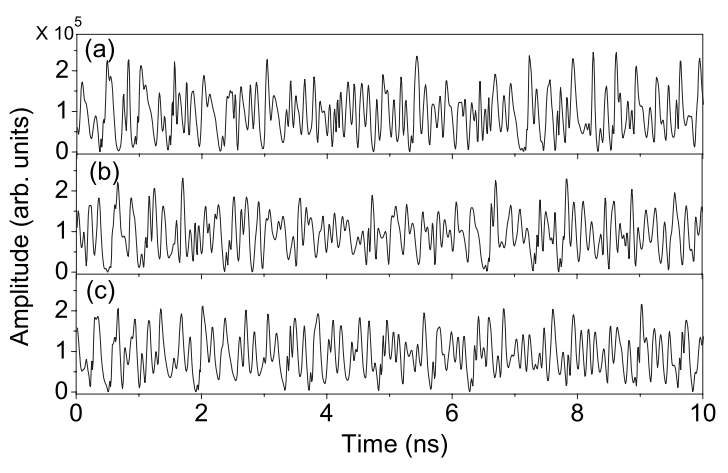

FIG. 3. Time series of the ML for different filter widths. (a) Without filter, (b) $\Lambda_{m} / 2 \pi=16 \mathrm{GHz}$ and (c) $\Lambda_{m} / 2 \pi=8 \mathrm{GHz}$. Parameters: $\gamma_{m}=25 \mathrm{~ns}^{-1}, I=1.5 I_{\mathrm{th}}$.

$$
A(t)=\left\langle\left[P_{m}\left(t^{\prime}\right) P_{m}\left(t^{\prime}-t\right)-\bar{P}_{m} \bar{P}_{m}\right]\right\rangle_{t^{\prime}} /\left(\sigma_{P_{m}} \sigma_{P_{m}}\right) .
$$

In Eq. (9) $\langle\cdots\rangle$ stands for the time average, and $\bar{P}_{m}$ and $\sigma_{P_{m}}$ are the mean and standard deviation of the ML output power.

In Fig. 4, we present the autocorrelation time as a function of the pump current and three filter widths. For the computation of this figure the filters are centered around $\omega_{m}$ $=0 \mathrm{GHz}$. We have checked that the results do not qualitatively change if we use a different central frequency. It can be seen that for all filter widths the autocorrelation time has a minimum (maximum complexity) around $I \approx 1.5 I_{\text {th }}$. This is in agreement with the fact that for conventional feedback the entropy computed from the Lyapunov exponents shows a maxima for $I \approx 1.5 I_{\text {th }}[28]$. This maximum appears due to the nonlinear saturation of the gain and takes place at larger values of $I$ when the gain saturation coefficient $s$ is reduced. For the saturation value considered here the entropy is maximum for $I$ around $1.5 I_{\text {th }}$ threshold, even in the case of filtered feedback [29]. It is also visible in Fig, 4 that the width of the filter modifies the complexity of the dynamics.

Throughout most of this paper we consider two filter widths -8 and $16 \mathrm{GHz}$ - that are slightly narrower than the total bandwidth at $I=1.5 I_{\text {th }}$ and conventional optical feedback. However, the bandwidth of the chaotic dynamics increases with increasing current. At currents above $1.5 I_{\text {th }}$, these filters reduce the complexity of the ML dynamicsincreasing the autocorrelation time-since they restrict the dynamical evolution of the system. It is known that the ad-

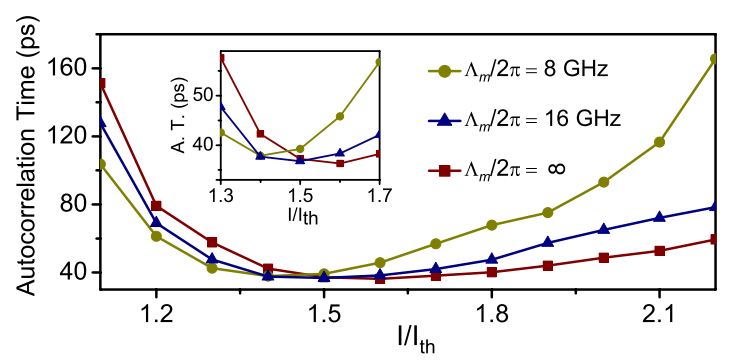

FIG. 4. (Color online) Autocorrelation time of the ML for different filter widths vs current. The inset is an enlargement of the autocorrelation time around $I / I_{\mathrm{th}}=1.5$.

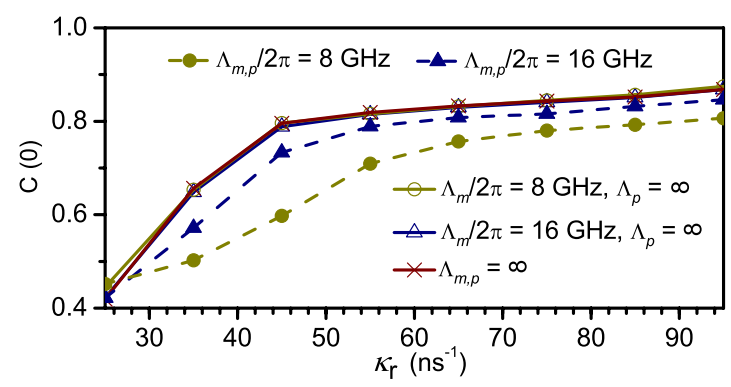

FIG. 5. (Color online) Isochronous correlation of ML and SL in the open-loop scheme for increasing coupling strength, and three different ML filter widths, when the output of the ML is sent directly to the SL (full lines) and when it is further filtered before it is injected into the SL (dashed lines). Parameters: $\gamma_{m}=25 \mathrm{~ns}^{-1}, \omega_{m, p}$ $=-8 \mathrm{GHz}, \gamma_{s}=0, I=1.5 I_{\mathrm{th}}$.

dition of a filter in the external cavity can reduce the number of external cavity modes and change their position, modifying the trajectories of the dynamics [18]. For very narrow filters, compared to the total bandwidth of the dynamics, the emission is restricted to limit cycle oscillations or even steady state [19]. At currents below $1.5 I_{\text {th }}$, the optical spectra are narrower than the filter bandwidths, while the filters are not centered at the maxima of the optical spectra. As a result, the filter destabilizes the dynamics of the filtered feedback systems at low currents, leading to short autocorrelation times.

\section{OPEN-LOOP SCHEME}

We start by discussing the numerical results obtained for the open-loop configuration. Here, we study two possibilities. In the more classical approach, the output of the ML is directly coupled into the SL. We also suggest a new configuration for the open loop in the case of filtered feedback, where the output of the ML is sent to a similar grating as the one in the feedback loop before it is coupled into the SL.

To measure the synchronization we compute the normalized cross correlation between the master and slave output powers, defined as

$$
C(t)=\left\langle\left[P_{m}\left(t^{\prime}\right) P_{s}\left(t^{\prime}-t\right)-\bar{P}_{m} \bar{P}_{s}\right]\right\rangle_{t^{\prime}} /\left(\sigma_{P_{m}} \sigma_{P_{s}}\right),
$$

where $\bar{P}_{m, s}\left(\sigma_{P_{m, s}}\right)$ are the mean (standard deviation) of the ML and SL output powers, respectively. Two different types of synchronization are usually considered in these systems: the isochronous synchronization, comparing $E_{m}(t)$ with $E_{s}(t)$, and the achronal one, comparing $E_{m}(t)$ with $E_{s}(t-\tau)$, which is found when the coupling and feedback parameters satisfy the condition $\gamma_{m}=\kappa_{r}+\gamma_{s}$ [30].

Figure 5 shows the isochronous cross correlation of ML and SL for $I=1.5 I_{\mathrm{th}}$. The cross-correlation coefficients never reach values above 0.9 even for large coupling strengths. The solid line with crosses corresponds to the conventional feedback case, i.e., without any filter. The addition of a filter in the feedback loop of the ML does not change the correlation between ML and SL, i.e., the solid lines with triangles and circles overlap in Fig. 5. Furthermore, the addition of a grat- 


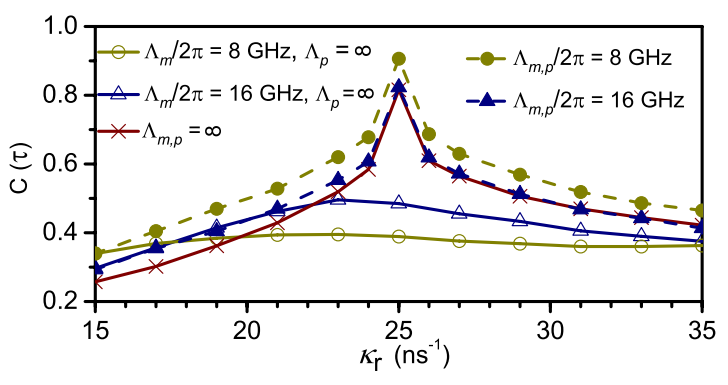

FIG. 6. (Color online) Correlation at $t=\tau$ of ML and SL in the open-loop scheme for increasing coupling strength, and three different ML filter widths, when the output of the ML is sent directly to the SL (full lines) and when it is further filtered before it is injected into the SL (dashed lines). Parameters: see Fig. 5.

ing in front of the SL decreases the isochronous correlation since the SL tries to synchronize to the filtered version of the ML; see the dashed lines with triangles and circles in Fig. 5. The low values of the correlation for the isochronous synchronization in the case of conventional feedback and open loop coincide with the results reported in [7], where the achronal synchronization leads to higher correlations.

Figure 6 shows $C(\tau)$-achronal synchronization-as a function of the coupling for $I=1.5 I_{\mathrm{th}}$. For conventional feedback (solid line with crosses) the correlation shows a sharp peak at $\kappa_{r}=\gamma_{m}$, which comes from the fact that for these precise values of the parameters there is a mathematical solution of the system satisfying $E_{s}(t-\tau)=E_{m}(t)$ [30]. Here identical synchronization $C(\tau)=1$ is not achieved because the achronal synchronization is unstable at $I=1.5 I_{\text {th }}[8]$. Achronal synchronization is stable for other pump currents where $C(\tau)=1$ is obtained. When considering filtered optical feedback and with no extra grating placed in front of the SL, $E_{s}(t-\tau)=E_{m}(t)$ is no longer a solution of the system for any value of the coupling, therefore the sharp peak at $\kappa_{r}=\gamma_{m}$ disappears and the synchronization is poor for any value of the coupling (solid lines with triangles and circles in Fig. 6). The addition of an extra grating in front of the SL allows the existence of a mathematical solution of the form $E_{s}(t-\tau)$ $=E_{m}(t)$ for $\kappa_{r}=\gamma_{m}$. In fact, Eqs. (2) and (3) are identicalwith a lag $\tau$-to Eqs. (4) and (5) for $\kappa_{r}=\gamma_{m}, \Lambda_{p}=\Lambda_{m}$, and $\omega_{p}=\omega_{m}$ so that $F_{p}(t)$ is the delayed version of $F_{m}(t)$. In other words, the input field in the SL plays the same role as the feedback in the ML, allowing for the existence of a solution of the form $E_{s}(t-\tau)=E_{m}(t)$. The narrow synchronization peak at $\kappa_{r}=\gamma_{m}$ is recovered and in fact synchronization is slightly improved with respect to the conventional feedback case (dashed lines with triangles and circles in Fig. 6).

A narrow filter improves the achronal correlation since the filter discriminates high frequencies which, in general, are known to synchronize worse. The synchronization also depends on the complexity of the dynamics of the ML. As shown in the inset of Fig. 4, the dynamics of the ML is slightly less complex for a filter width of $\Lambda_{m} / 2 \pi=8 \mathrm{GHz}$ than for a filter width of $\Lambda_{m} / 2 \pi=16 \mathrm{GHz}$. The reduction in complexity together with the filtering of high frequencies leads to a better achronal synchronization of filtered feedback systems in the open-loop configuration.

To finish the discussion of the results for the open-loop configuration, we evaluate the achronal synchronization of

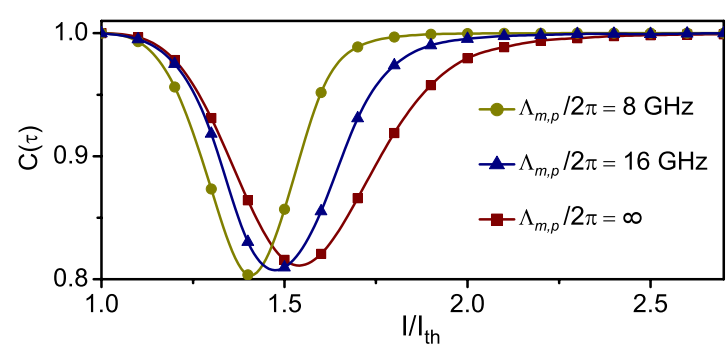

FIG. 7. (Color online) Correlation at $t=\tau$ of ML and SL in the open-loop scheme for increasing injection current $I$ and three different ML filter widths. Parameters: $\gamma_{m}=25 \mathrm{~ns}^{-1}, \omega_{m, p}=0 \mathrm{GHz}, \gamma_{s}$ $=0, \kappa_{r}=25 \mathrm{~ns}^{-1}$.

the ML and SL for increasing injection current and three different filter widths. Although the maximum of the optical spectrum moves when the injection current is increased, for simplicity, we keep the filters always centered at $0 \mathrm{GHz}$. We must stress again that the choice of another central frequency for the filter does not change the results qualitatively. It can be seen in Fig. 7 that the correlations have a value close to 1 for small and for large currents but show a minimum around $I=1.5 I_{\text {th }}$. This minimum coincides with the minimum of the autocorrelation time shown in Fig. 4. The achronal solution is an exact solution of the system but it is not always stable. When it is stable the open-loop configuration provides identical synchronization between the ML and SL. However, when it is not stable the synchronization degrades. Similar results were obtained in conventional optical feedback schemes [8].

\section{CLOSED-LOOP SCHEME}

We now focus our attention on the closed-loop configuration. In this scheme, achronal synchronization occurs when $\gamma_{m}=\kappa_{r}+\gamma_{s}$. However, in practice, a high degree of synchronization is obtained only for very low values of the feedback strength of the SL $\left(\gamma_{s}\right)[7]$, which is comparable to the openloop system that we have already studied. Consequently, we show results only for the isochronous solution in the closedloop scheme.

Figure 8 shows the isochronous correlation coefficient for different filter widths as a function of the coupling strength. Filters are centered at $-8 \mathrm{GHz}$ corresponding to the maxima

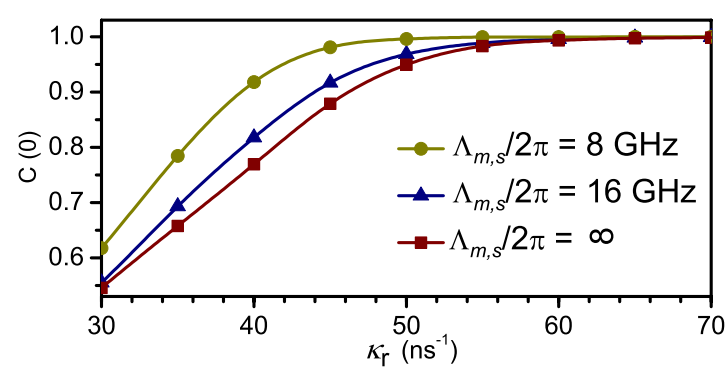

FIG. 8. (Color online) Synchronization of ML and SL in the closed-loop scheme for increasing coupling strength and three different filter widths. Parameters: $\gamma_{m}=\gamma_{s}=25 \mathrm{~ns}^{-1}, \Lambda_{s}=\Lambda_{m}, \omega_{s}=\omega_{m}$ $=-8 \mathrm{GHz}, I=1.5 I_{\text {th }}$. 


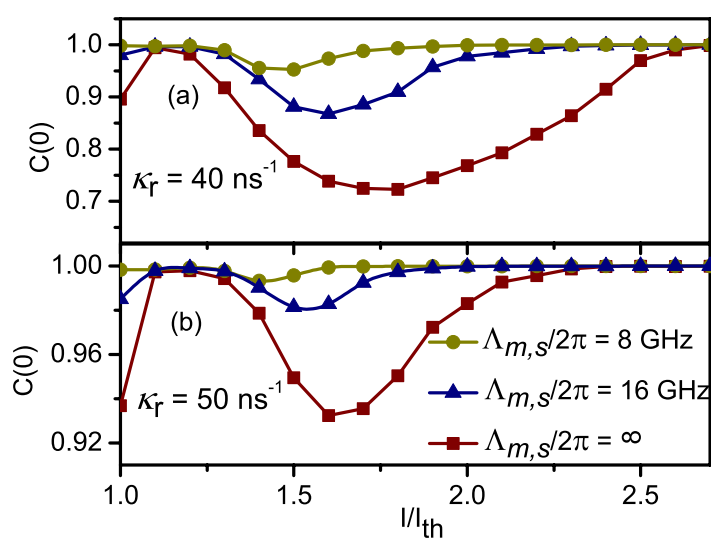

FIG. 9. (Color online) Correlation at $t=0$ of ML and SL in the closed-loop scheme for increasing injection current $I$ and three different ML filter widths. Parameters: $\omega_{s}=\omega_{m}=0 \mathrm{GHz}, \kappa_{r}=($ a) 40 and (b) $50 \mathrm{~ns}^{-1}$. Other parameters as in Fig. 8 .

of the optical spectra when $I=1.5 I_{\text {th }}$. For large enough coupling $\left(\kappa_{r}>55 \mathrm{~ns}^{-1}\right)$ excellent synchronization is obtained for any filter width. Furthermore, on narrowing the filter widths, a correlation close to 1 is achieved for smaller coupling values. We stress that, for the values of the filter widths that we present, the evolution of the system is still in a chaotic state. In general, we observe larger cross correlations because the filter discriminates high frequencies that are known to deteriorate the synchronization. The improvement in the correlation obtained for the narrowest filter $(8 \mathrm{GHz})$ can also be attributed to the fact that with this filter the autocorrelation time of the chaotic wave form slightly increases, as shown in the inset of Fig. 4.

The closed-loop configuration gives maximal synchronization for a wider range of parameters than its open-loop counterpart provided that the feedback arms are perfectly matched in the ML and SL. Furthermore, the closed-loop configuration provides maximal synchronization at any injected current $I$ with the condition that the coupling is adjusted accordingly.

The synchronization between ML and SL depends on the injection current. Figures 9(a) and 9(b) show the isochronous cross correlation versus current for two coupling strengths $\kappa_{r}=40$ and $50 \mathrm{~ns}^{-1}$. The synchronization improves when the coupling increases [note the different scale of the $y$ axis in Figs. 9(a) and 9(b)]. The correlation has a minimum at the currents where the autocorrelation time is minimum (see Fig. 4). Away from the minimum, $C(0) \approx 1$ for large and small currents except very close to the laser threshold where the system exhibits low-frequency fluctuations (LFFs) [31]. The LFF dynamics is characterized by power dropouts followed by a slow recovery until the next power dropout. The cross correlation between ML and SL degrades because the synchronization is momentarily lost after each power dropout. This effect is not found in the open loop since the SL is subject to optical injection but is not simultaneously subject to optical feedback. It is clear from Fig. 9 that filtered feedback systems require less coupling to achieve a good performance. If the coupling strength is increased $\left(\kappa_{r}>60 \mathrm{~ns}^{-1}\right)$ then $C(0)=1$ for any current and any filter bandwidth.

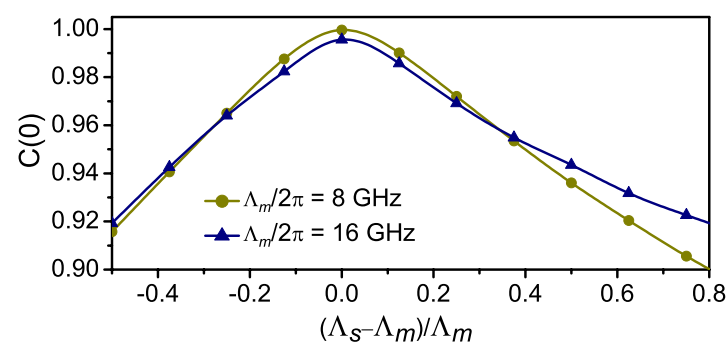

FIG. 10. (Color online) Synchronization of ML and SL in the closed-loop scheme for a mismatch in the width of the filters in ML and SL. Parameters: $\gamma_{m}=\gamma_{s}=25 \mathrm{~ns}^{-1}, \quad \kappa_{r}=60 \mathrm{~ns}^{-1}, \omega_{s}=\omega_{m}, I$ $=1.5 I_{\mathrm{th}}$.

We have also checked the robustness of the synchronized solutions under the presence of spontaneous emission noise. When Langevin noise sources with realistic values are added to Eqs. (2)-(7), the isochronous correlation degrades by less than $1 \%$ in the closed-loop configuration, while the achronal synchronization degrades by $5-10 \%$ in the open-loop configuration. This demonstrates again the high sensibility of the achronally synchronized solution.

Finally, we check the influence of a mismatch in the parameters of the filters in the ML and SL. Here, we keep the parameters of the filter in the ML constant and vary those of the filter in the SL. Figures 10 and 11 show how the correlation decays when the filters in the ML and SL are slightly different.

For instance, we have checked that a value of $C>0.95$ can be reached for a range of SLs filters from $\sim 12$ to $\sim 22 \mathrm{GHz}$ or when the filter central frequencies differ by $\sim 2 \mathrm{GHz}$ for a filter of $\Lambda_{m} / 2 \pi=16 \mathrm{GHz}$ and coupling $\kappa_{r}=60 \mathrm{~ns}^{-1}$.

\section{RECEIVER RESPONSE TO A PERTURBATION: INJECTION LOCKING VS SYNCHRONIZATION}

In this section, we focus on the influence of adding a perturbation, in the form of a binary sequence of bits, to the output of the master laser. In general terms, when the SL synchronizes to the ML it reproduces the chaotic dynamics of the ML and filters the perturbation, i.e., the encoded message. On the contrary, when the SL locks to the ML, the latter reproduces the incoming field, which is a combination of the chaotic carrier and the embedded message. We will

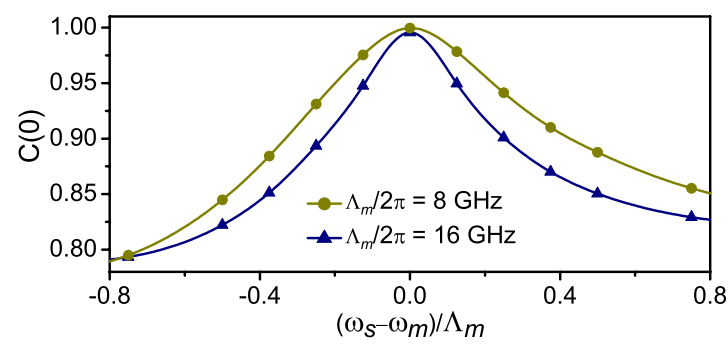

FIG. 11. (Color online) Synchronization of ML and SL in the closed-loop scheme for a mismatch in the central frequency of the filters in ML and SL. Parameters: $\gamma_{m}=\gamma_{s}=25 \mathrm{~ns}^{-1}, \kappa_{r}=60 \mathrm{~ns}^{-1}$, $\Lambda_{s}=\Lambda_{m}, I=1.5 I_{\mathrm{th}}$. 


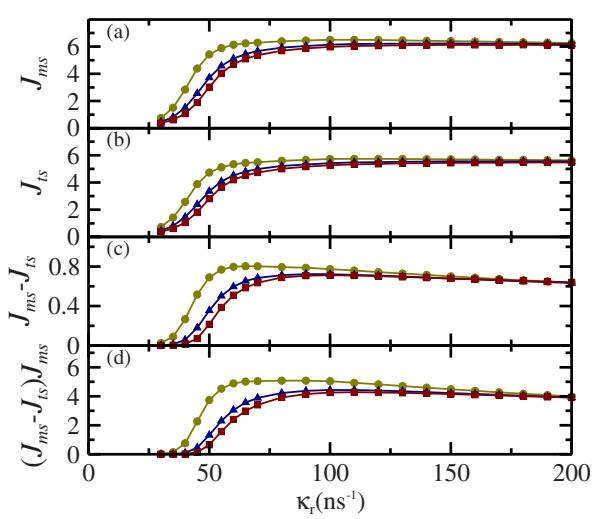

FIG. 12. (Color online) Average mutual information in the closed-loop scheme vs coupling strength for three different filter widths at $I=1.5 I_{\text {th }} . \bullet, \Lambda / 2 \pi=8 \mathrm{GHz}, \boldsymbol{\Delta}, \Lambda / 2 \pi=16 \mathrm{GHz}, \boldsymbol{\square}, \Lambda$ $=\infty$. Parameters: $\epsilon=0.05$. Other parameters as in Fig. 8 .

show that synchronization typically occurs for a relatively weak coupling and as the coupling strength increases there is a continuous transition to the injection-locking regime.

The scheme we choose to encode information is chaotic modulation. In this scheme, the message (perturbation) is added by modulating the transmitter's chaotic carrier, according to the expression $P_{t}(t)=[1-\epsilon m(t)] P_{m}(t)$ [32], where $\epsilon$ is the amplitude of the modulation and $m(t)$ is the message being transmitted. The message can be recovered at the receiver side as follows: $m^{\prime}(t)=\left[1-P_{t}(t) / P_{s}(t)\right] / \epsilon$. The extraction of the message is possible if the slave laser reproduces mainly the chaotic carrier. For $P_{s}(t)=P_{m}(t)$ (ideal synchronization), the message is perfectly recovered. For $P_{s}(t)=P_{t}(t)$ (perfect locking), the message is not recovered.

A useful tool to distinguish between chaos synchronization and injection locking is the average mutual information. This nonlinear measure of the similarities between two quantities $x, y$ is defined as [33]

$$
J_{x y}=\sum_{i, j} p_{i j} \log _{2} \frac{p_{i j}}{p_{i} p_{j}},
$$

where $p_{i j}$ is the joint probability of $x=x_{i}$ and $y=y_{j}, p_{i}$ is the probability of $x=x_{i}$ and $p_{j}$ is the probability of $y=y_{j}$. This quantity essentially measures the extra information one gets from a signal when the outcome of the other signal is known. Thus, if there is no connection between the two signals $p_{i j}$ $=p_{i} p_{j}$, and $J_{x y}$ is zero. Otherwise, $J_{x y}$ will be positive, taking its maximum value for identical signals. Here we compute the average mutual information between the optical power of the $\operatorname{ML}\left[P_{m}(t)\right]$ and $\operatorname{SL}\left[P_{s}(t)\right]$ denoted as $J_{m s}$ and the average mutual information between the transmitted signal $P_{t}(t)$ and the slave signal $P_{s}(t)\left(J_{t s}\right)$. Both quantities $J_{m s}$ and $J_{t s}$ are evaluated when a message is codified in the output of the ML. In the synchronization regime $J_{m s}>J_{t s}$ so that the receiver filters out the message. In the injection-locking regime $J_{t s}>J_{m s}$.

For the sake of clarity, we will first discuss the closedloop configuration. Figure 12 shows $J_{m s}$ [Fig. 12(a)] and $J_{t s}$ [Fig. 12(b)] when a message with amplitude $\epsilon=0.05$ is encoded in the output of the ML.
For very low coupling, the dynamics of ML and SL is uncorrelated and therefore the mutual information vanishes. On increasing the coupling $J_{m s}$ increases, indicating a large correlation between ML and SL outputs, reaching a plateau for large enough couplings. The plateau of maximum average mutual information is reached for a coupling larger than the one for which maximum correlation is achieved in Fig. 8. This is because the correlation shown in Fig. 8 corresponds to the direct injection of the ML into the SL without any added message. When the message is encoded, a slightly larger coupling is needed in order to achieve maximum correlation. For intermediate values of the coupling, the effect of filtering the feedback is to increase the synchronization between ML and SL, in agreement with the correlation results shown in Fig. 8.

Since the amplitude of the message is small, the injected signal is in fact quite correlated to the carrier. Therefore, the average mutual information between the injected signal and the response of the SL, $J_{t s}$ [Fig. 12(b)], shows a similar behavior to $J_{m s}$. Nevertheless, $J_{m s}$ is clearly larger than $J_{t s}$ as shown in Fig. 12(c). This means that, under these conditions, synchronization between the SL and ML prevails over injection locking. However, for large couplings, the difference between $J_{m s}$ and $J_{t s}$ decreases as the coupling is increased. We have checked that injection locking dominates over chaos synchronization for $\kappa_{r}>1000 \mathrm{~ns}^{-1}$, which is quite beyond the physical range of couplings.

In chaos-based communications one requires a large synchronization between the output of the ML and SL for the optimal recovery of a message, namely, a large value of $J_{m s}$, and also a large discrimination, i.e., a large value of $J_{m s}$ $-J_{t s}$. Therefore, we propose to use the product $J_{m s}\left(J_{m s}-J_{t s}\right)$ as an indicator of the receiver quality, that is, the capability of recovering the message. As shown in Fig. 12(d), closedloop receivers are very good at recovering messages for a quite large range of coupling strengths. Filtering the feedback increases the recovering capabilities.

Increasing the injection current from $I=1.5 I_{\text {th }}$ to $2 I_{\text {th }}$ leads to a less chaotic output of the ML, as indicated by the larger autocorrelation time shown in Fig. 4. In correspondence, the average mutual information at $I=2 I_{\text {th }}$ is larger than at $I$ $=1.5 I_{\text {th }}$, as shown in Fig. 13. The difference $\left(J_{m s}-J_{t s}\right)$ also increases at this current, as can be seen in Fig. 13(c). The results for the filter of width $\Lambda / 2 \pi=8 \mathrm{GHz}$ are not shown, since the time trace is much less chaotic (see the large autocorrelation time shown Fig. 4 for this filter at this current).

Figure 14 shows the transmitted message and the recovered message for two different coupling strengths. The extracted message is filtered with a third-order Butterworth low-pass filter. The cutoff frequency of the filter is set to 0.75 times the modulation bit rate of the message, which is $1 \mathrm{Gbit} / \mathrm{s}$ in this example. It can be seen in Figs. 14(b) and 14(c) that the message can be extracted only when $J_{m s}$ and the difference $J_{m s}-J_{t s}$ are sufficiently large.

The situation is quite different in the open loop configuration (Fig. 15). The difference between $J_{m s}$ and $J_{t s}$ is now much smaller, so the distinction between synchronization and injection locking is weaker. In fact, for a message amplitude like the one we considered in the closed loop, the message cannot be practically recovered at the receiver. Con- 


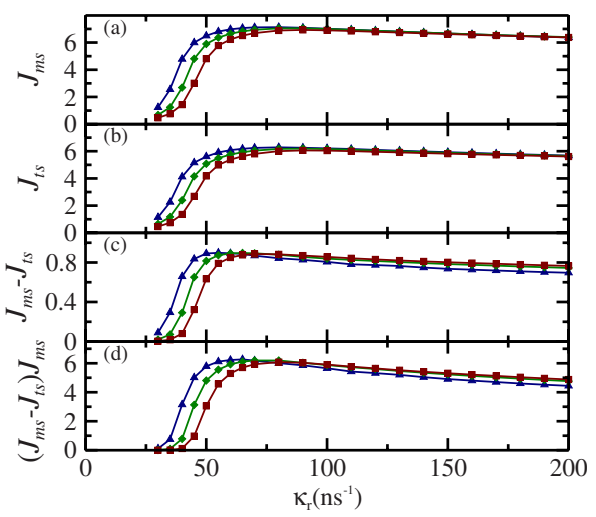

FIG. 13. (Color online) Mutual information of ML (with and without message) and SL in the closed-loop scheme vs coupling strength for three different filter widths at $I=2 I_{\mathrm{th}} . \quad \boldsymbol{\Delta}, \Lambda / 2 \pi$ $=16 \mathrm{GHz}, \diamond, \Lambda / 2 \pi=30 \mathrm{GHz}, \boldsymbol{\square}, \Lambda=\infty$. Other parameters as in Fig. 12.

sequently, for the open loop we consider a much larger message amplitude $\epsilon=0.15$. We also consider only the case $I$ $=2 I_{\text {th }}$ where the system is less chaotic. Despite all that, the difference $J_{m s}-J_{t s}$ is much smaller than in the closed loop, as shown in Fig. 15(c). The difference first increases with the coupling strength reaching a maximum at $\kappa_{r} \approx 400 \mathrm{~ns}^{-1}$, quite beyond realistic values, after which it decreases and at $k_{r} \approx 1300 \mathrm{~ns}^{-1}$ (not shown in the figure) it becomes negative, indicating that the system enters in the injection-locking regime. Even where the difference $J_{m s}-J_{t s}$ is maximum, it only takes the value 0.4 , less than one-half of the maximum obtained for a closed loop using a message with only one-third of the amplitude. Furthermore, the average mutual information $J_{m s}$ is also much smaller than for closed-loop receivers. This is in agreement with the synchronization obtained between the ML and SL even in the absence of a message and shown in Fig. 5. Therefore, the receiver quality indicator $J_{m s}\left(J_{m s}-J_{t s}\right)$ is considerably smaller than for a closed loop [notice the different vertical scale in Fig. 15(d) as compared with Fig. 13(d)].

Figure 16 shows the transmitted and recovered messages for two different coupling strengths in the open-loop configuration. There, it can be seen that the message is recovered only if large couplings such as $\kappa_{r}=150 \mathrm{~ns}^{-1}$, which would

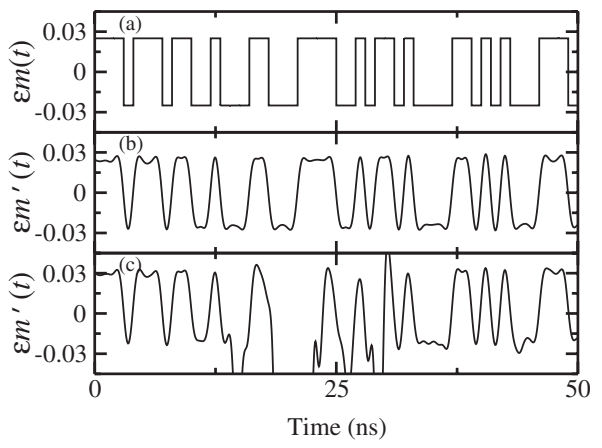

FIG. 14. (a) Transmitted message. Message recovery in the closed loop for $\kappa_{r}=70$ (b) and (c) $45 \mathrm{~ns}^{-1}$. Parameters: $\Lambda / 2 \pi$ $=16 \mathrm{GHz}$. Other parameters as in Fig. 13 .

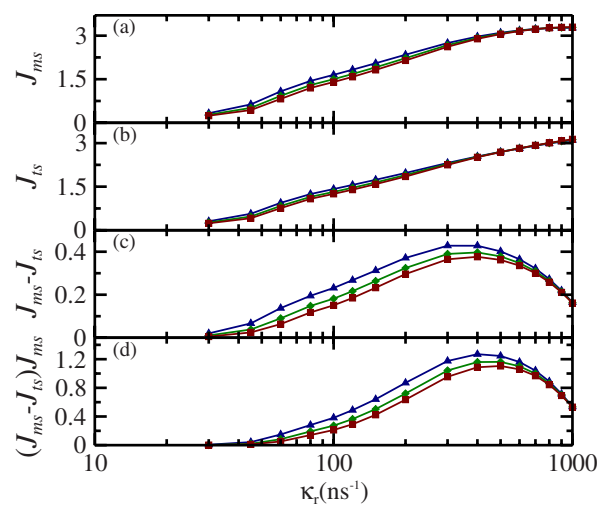

FIG. 15. (Color online) Mutual information of ML (with and without message) and SL in the open-loop scheme vs coupling strength for three different filter widths at $I=2 I_{\text {th }} . \boldsymbol{\Delta}, \Lambda / 2 \pi$ $=16 \mathrm{GHz}, \diamond, \Lambda / 2 \pi=30 \mathrm{GHz}, \boldsymbol{\square}, \Lambda=\infty$. Parameters: $\gamma_{s}=0, \epsilon$ $=0.15$. Other parameters as in Fig. 13 .

require external amplification, are considered. And even for these parameters the quality of the recovered message is not as good as those obtained with closed-loop receivers with much smaller couplings and using smaller message amplitudes.

\section{CONCLUSION}

We have shown that the chaos synchronization extensively studied for SCLs subject to feedback is still maintained when filtered feedback is considered. By introducing a filter in the feedback loop, we have added two extra control parameters in the system, i.e., the filter width and central frequency, that could be used to improve privacy in chaotic communication systems. The slight reduction in complexity together with the filtering of high frequencies leads to a better performance-higher cross correlation between the ML and SL-in the closed-loop scheme. In fact, closed-loop receivers show very good isochronous synchronization even when the parameters of the ML and SL, including those corresponding to the filters, have a small mismatch. The computation of the average mutual information indicates that closed-loop receivers are very good at discriminating between the chaotic carrier and the transmitted signal (carrier

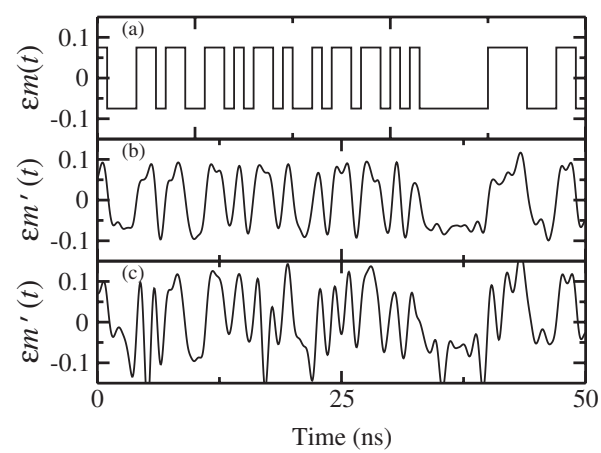

FIG. 16. (a) Transmitted message. Message recovery in the open loop for $\kappa_{r}=150$ (b) and (c) $60 \mathrm{~ns}^{-1}$. Parameters: $\Lambda / 2 \pi=16 \mathrm{GHz}$. Other parameters as in Fig. 15. 
with message) even for small message amplitudes. This allows a good extraction of the message even for relatively small coupling strengths. The filtered feedback enhances the discrimination capability and improves the quality of the recovered message.

On the contrary, the performance of open-loop receivers is rather limited. The isochronous solution gives poor synchronization when filtered feedback is used and for the achronal synchronization the correlation only reaches a value close to 1 for a limited range of injected currents and with a coupling coefficient that precisely matches the ML feedback strength. Furthermore, the computation of the average mutual information shows that the differentiation between the chaotic carrier and the transmitted signal achieved with open loop receivers is also limited. Large-amplitude messages together with large coupling amplitudes are required to recover the, message. This indicates that when conventional or filtered optical feedback is used, the use of a closed-loop receiver clearly yields a higher synchronization degree and a better message extraction.

The addition of a binary sequence of bits to the ML output and the corresponding extraction of this message using the SL output allows us to distinguish between chaos synchronization and injection locking. The computation of the mutual information indicates that chaos synchronization dominates over injection locking for a wide range of coupling strengths.

\section{ACKNOWLEDGMENTS}

The authors acknowledge financial support from MEC (Spain) and Feder under Project Nos. TEC2006-1009/MIC (PhoDECC), TEC-2006-28105-E, and FIS2007-60327 (FISICOS); from EC Project PICASSO Grant No. IST-200534551, and from CONACYT (Mexico) Project No. 46973. M.C.S. thanks the MEC for a "Juan de la Cierva" scientific contract. We are grateful to Dr. Kostas Chlouverakis for helpful discusions.
[1] C. R. Mirasso, P. Colet, and P. García-Fernández, IEEE Photonics Technol. Lett. 8, 299 (1996).

[2] IEEE J. Quantum Electron. 38, 1138 (2002), special issue on optical chaos and applications to cryptography, edited by $\mathrm{S}$. Donati and C. R. Mirasso.

[3] C. R. Phys. 5, 609 (2004), special issue on cryptography using optical chaos, edited by L. Larger and J. P. Goedgebuer.

[4] A. Uchida, F. Rogister, J. Garcia-Ojalvo, and R. Roy, Prog. Opt. 48, 203 (2005).

[5] A. Argyris, D. Syvridis, L. Larger, V. Annovazzi-Lodi, P. Colet, I. Fischer, J. García-Ojalvo, C. R. Mirasso, L. Pesquera, and K. A. Shore, Nature (London) 438, 343 (2005).

[6] M. Yousefi, Y. Barbarin, S. Beri, E. A. J. M. Bente, M. K. Smit, R. Nötzel, and D. Lenstra, Phys. Rev. Lett. 98, 044101 (2007).

[7] R. Vicente, T. Pérez, and Claudio R. Mirasso, IEEE J. Quantum Electron. 38, 1197 (2002).

[8] A. Locquet, C. Masoller, and C. R. Mirasso, Phys. Rev. E 65, 056205 (2002).

[9] M. Peil, T. Heil, I. Fischer, and W. Elsässer, Phys. Rev. Lett. 88, 174101 (2002).

[10] T. Heil, J. Mulet, I. Fischer, C. R. Mirasso, M. Peil, P. Colet, and W. Elsässer, IEEE J. Quantum Electron. 38, 1162 (2002).

[11] V. Annovazzi-Lodi, S. Donati, and A. Scire, IEEE J. Quantum Electron. 32, 953 (1996).

[12] A. Argyris, D. Kanakidis, A. Bogris, and D. Syvridis, IEEE J. Sel. Top. Quantum Electron. 10, 927 (2004).

[13] T. Perez, M. Radziunas, H. J. Wünsche, Claudio R. Mirasso, and F. Henneberger, IEEE Photonics Technol. Lett. 18, 2135 (2006).

[14] F. Rogister, A. Locquet, D. Pieroux, M. Sciamanna, O. Deparis, P. Megret, and M. Blondel, Opt. Lett. 26, 1486 (2001).

[15] D. Sukow, K. Blackburn, A. Spain, K. Babcock, L. Bennett, and A. Gavrielides, Opt. Lett. 29, 2393 (2004).

[16] N. Gastaud, S. Poinsot, L. Larger, J. M. Merolla, M. Hanna, J. P. Goedgebuer, and E. Malassenet, Electron. Lett. 40, 898 (2004).

[17] M. Y. Kim, C. Sramek, A. Uchida, and R. Roy, Phys. Rev. E 74, 016211 (2006).

[18] M. Yousefi and D. Lenstra, IEEE J. Quantum Electron. 35, 970 (1999).

[19] A. P. A. Fischer, O. K. Andersen, M. Yousefi, S. Stolte, and D. Lenstra, IEEE J. Quantum Electron. 36, 375 (2000).

[20] L. M. Pecora and T. L. Carroll, Phys. Rev. Lett. 64, 821 (1990).

[21] R. Lang and K. Kobayashi, IEEE J. Quantum Electron. 16, 347 (1980).

[22] A. A. Duarte and H. G. Solari, Phys. Rev. A 58, 614 (1998).

[23] M. Giudici, L. Giuggioli, C. Green, and J. R. Tredicce, Chaos, Solitons Fractals 10, 811 (1999).

[24] M. Homar, S. Balle, and M. San Miguel, Opt. Commun. 131, 380 (1996).

[25] I. Fischer, G. H. M. van Tartwijk, A. M. Levine, W. Elsässer, E. Göbel, and D. Lenstra, Phys. Rev. Lett. 76, 220 (1996).

[26] T. Heil, I. Fischer, W. Elsässer, J. Mulet, and C. R. Mirasso, Opt. Lett. 24, 1275 (1999).

[27] C. Henry, IEEE J. Quantum Electron. 18, 259 (1982).

[28] R. Vicente, J. Daudén, P. Colet, and R. Toral, IEEE J. Quantum Electron. 41, 541 (2005).

[29] Kostas Chlouverakis (private communication).

[30] C. Masoller, Phys. Rev. Lett. 86, 2782 (2001).

[31] T. Sano, Phys. Rev. A 50, 2719 (1994).

[32] D. Kanakidis, A. Argyris, and D. Syvridis, J. Lightwave Technol. 21, 750 (2003).

[33] M. Paluš, Physica D 80, 186 (1995), and references therein. 